\title{
Positive solutions of $m$-point multi-term fractional integral BVP involving time-delay for fractional differential equations
}

\author{
Kaihong Zhao* and Ping Gong
}

\author{
"Correspondence: \\ zhaokaihongs@126.com \\ Department of Applied \\ Mathematics, Kunming University of \\ Science and Technology, Kunming, \\ Yunnan 650093, China
}

\begin{abstract}
In the paper, we establish sufficient conditions for the existence and multiplicity of positive solutions to a class of higher-order delayed nonlinear fractional differential equations with $m$-point multi-term fractional integral boundary conditions. The results are established by converting the problem into an equivalent integral equation and applying fixed point theorems of the cone expansion and compression of norm type. Our study improves the previous results in the literature. As an application, an example is also provided to illustrate our main results.
\end{abstract}

MSC: 34B10; 34B15; 34B18; 34B27

Keywords: fractional differential equations; multiple positive solutions; integral boundary value conditions; time-delay

\section{Introduction}

The fractional differential equation has a significant role to play in many fields such as physics, chemistry, aerodynamics, electrodynamics of a complex medium, polymer rheology, Bode's analysis of feedback amplifiers, capacitor theory, electrical circuits, electronanalytical chemistry, biology, control theory, fitting of experimental data, and so forth. The fractional differential equation also serves as an excellent tool for the description of hereditary properties of various materials and processes. In consequence, the subject of fractional differential equations is gaining much importance and attention. There are a large number of papers dealing with the existence or multiplicity of solutions or positive solutions of initial or boundary value problems for some differential equations. For details and examples, see [1-23] and the references therein. In [8, 9, 16, 18-23], the authors have discussed the existence of multiple positive solutions for boundary value problems of integer or fractional differential equations.

Integral boundary conditions have various applications in applied fields such as blood flow problems, chemical engineering, thermoelasticity, underground water flow, population dynamics, and so on. For more details of nonlocal and integral boundary conditions, see $[5,10-12,20]$ and the references therein. In addition, it is well known that delay arises naturally in practical systems due to the transmission of signal or the mechanical transmission. Though the theory of ordinary differential equations with delays is mature, not much has been done for fractional differential equations with delays; see [6,10,13, 14]. 
In [11], Tariboon et al. are concerned with the existence of at least one, two or three positive solutions for the boundary value problem with three-point multi-term fractional integral boundary conditions,

$$
\left\{\begin{array}{l}
D_{0+}^{q} u(t)+f(t, u(t))=0, \quad 1<q \leq 2, t \in[0,1] \\
u(0)=0, \quad u(1)=\sum_{i=1}^{m} \alpha_{i}\left(I_{0+}^{p_{i}} u\right)(\eta), \quad 0<\eta<1
\end{array}\right.
$$

where $f:[0,1] \times[0, \infty) \rightarrow[0, \infty) . D_{0+}^{q}$ is the standard Riemann-Liouville derivative of order $q . I_{0+}^{p_{i}}$ is the Riemann-Liouville fractional integral of order $p_{i}>0 . \alpha_{i} \geq 0(i=1,2, \ldots$, $m-2$ ) are real constants.

To the best of our knowledge, no one has studied the existence of multiple positive solutions with delayed nonlinear fractional differential equations. In this article, motivated by the above-mentioned papers, we study the existence of multiple positive solutions for the following higher-order delayed nonlinear fractional differential equation with $m$-point multi-term fractional integral boundary conditions:

$$
\left\{\begin{array}{l}
D_{0+}^{q} u(t)+f\left(t, u\left(t-\tau_{1}\right), u\left(t+\tau_{2}\right)\right)=0, \quad t \in[0,1] \\
u^{(j)}(0)=0, \quad u(1)=\sum_{i=1}^{m-2} \alpha_{i}\left(I_{0+}^{p_{i}} u\right)\left(\zeta_{i}\right), \quad 0 \leq j \leq n-2,0<\zeta_{i}<1
\end{array}\right.
$$

subject to the following initial conditions:

$$
\begin{cases}a u(t)-b u^{\prime}(t)=\eta(t), & t \in\left[-\tau_{1}, 0\right] \\ c u(t)+d u^{\prime}(t)=\xi(t), & t \in\left[1,1+\tau_{2}\right]\end{cases}
$$

where $0 \leq \tau_{1}, \tau_{2}<\theta \in(0,1 / 2)$ are suitably small. $D_{0^{+}}^{q}$ is the standard Riemann-Liouville derivative of order $n-1<q \leq n, n \geq 3 . a, b, c, d \geq 0, \eta(t) \in C\left(\left[-\tau_{1}, 0\right],[0,+\infty)\right), \xi(t) \in$ $C\left(\left[1,1+\tau_{2}\right],[0,+\infty)\right), f \in C([0,1] \times[0,+\infty) \times[0,+\infty),[0,+\infty)) . I_{0+}^{p_{i}}$ is the RiemannLiouville fractional integral of order $p_{i} . \alpha_{i} \geq 0(i=1,2, \ldots, m-2)$ are real constants such that $\sum_{i=1}^{m-2} \frac{\alpha_{i} \zeta_{i}^{p_{i}+q-1} \Gamma(q)}{\Gamma\left(p_{i}+q\right)}<1$.

Remark 1.1 By (1.2) and (1.3), we have $\eta(0)=0$ and $\xi(1)=c u(1)+d u^{\prime}(1)$.

Remark 1.2 When $1<q \leq 2, \tau_{1}=\tau_{2}=0, \varsigma_{i} \equiv \eta, j=0$, then (1.2) and (1.3) degenerate into (1.1). So our models extend (1.1).

\section{Preliminaries}

For the convenience of the reader, we present here the necessary definitions from fractional calculus theory. These definitions and properties can be found in the literature.

Definition 2.1 (see [24, 25]) The Riemann-Liouville fractional integral of order $\alpha>0$ of a function $u:(0, \infty) \rightarrow \mathbb{R}$ is given by

$$
I_{0+}^{\alpha} u(t)=\frac{1}{\Gamma(\alpha)} \int_{0}^{t}(t-s)^{\alpha-1} u(s) d s
$$

provided that the right-hand side is pointwise defined on $(0, \infty)$. 
Definition 2.2 (see [24, 25]) The Riemann-Liouville fractional derivative of order $\alpha>0$ of a continuous function $u:(0, \infty) \rightarrow \mathbb{R}$ is given by

$$
D_{0+}^{\alpha} u(t)=\frac{1}{\Gamma(n-\alpha)} \frac{d^{n}}{d t^{n}} \int_{0}^{t}(t-s)^{n-\alpha-1} u(s) d s
$$

where $n-1<\alpha \leq n$, provided that the right-hand side is pointwise defined on $(0, \infty)$.

Lemma 2.1 (see [17]) Assume that $u \in C(0,1) \cap L(0,1)$ with a fractional derivative of order $\alpha>0$ that belongs to $u \in C(0,1) \cap L(0,1)$. Then

$$
I_{0+}^{\alpha} D_{0+}^{\alpha} u(t)=u(t)+C_{1} t^{\alpha-1}+C_{2} t^{\alpha-2}+\cdots+C_{n} t^{\alpha-n},
$$

for some $C_{i} \in \mathbb{R}, i=1,2, \ldots, n$, where $n$ is the smallest integer greater than or equal to $\alpha$.

Lemma 2.2 (see [26]) Let $E$ be a Banach space, $P \subseteq E$ is a cone, and $\Omega_{1}, \Omega_{2}$ are two bounded open balls of $E$ centered at the origin with $0 \in \Omega_{1}$ and $\bar{\Omega}_{1} \subset \Omega_{2}$. Suppose that $A: P \cap\left(\bar{\Omega}_{2} \backslash \Omega_{1}\right) \rightarrow P$ is a completely continuous operator such that either

(i) $\|A u\| \leq\|u\|, u \in P \cap \partial \Omega_{1}$, and $\|A u\| \geq\|u\|, u \in P \cap \partial \Omega_{2}$, or

(ii) $\|A u\| \geq\|u\|, u \in P \cap \partial \Omega_{1}$, and $\|A u\| \leq\|u\|, u \in P \cap \partial \Omega_{2}$

holds. Then $A$ has at least one fixed point in $P \cap\left(\bar{\Omega}_{2} \backslash \Omega_{1}\right)$.

For simplicity, we put

$$
\Omega=1-\sum_{i=1}^{m-2} \frac{\alpha_{i} \zeta_{i}^{p_{i}+q-1} \Gamma(q)}{\Gamma\left(p_{i}+q\right)} .
$$

Now we present the Green's function for BVP (1.2)-(1.3).

Lemma 2.3 Let $\sum_{i=1}^{m-2} \frac{\alpha_{i} \zeta_{i}^{p_{i}+q-1} \Gamma(q)}{\Gamma\left(p_{i}+q\right)}<1, \alpha_{i} \geq 0, p_{i}>0(i=1,2, \ldots, m-2)$, and $h \in C([0,1], \mathbb{R})$, $1 \leq n-1<q \leq n$. The unique solution of

$$
\left\{\begin{array}{l}
D_{0+}^{q} u(t)+h(t)=0, \quad t \in[0,1], \\
u^{(j)}(0)=0, \quad u(1)=\sum_{i=1}^{m-2} \alpha_{i}\left(I_{0+}^{p_{i}} u\right)\left(\zeta_{i}\right), \quad j=0,1, \ldots, n-2,0<\zeta_{i}<1,
\end{array}\right.
$$

is

$$
u(t)=\int_{0}^{1} G(t, s) h(s) d s,
$$

where $G(t, s)$ is the Green's function given by

$$
G(t, s)=g(t, s)+\sum_{i=1}^{m-2} \frac{\alpha_{i} t^{q-1}}{\Omega \Gamma\left(p_{i}+q\right)} g_{i}\left(\zeta_{i}, s\right),
$$

where

$$
g(t, s)=\frac{1}{\Gamma(q)} \begin{cases}t^{q-1}(1-s)^{q-1}-(t-s)^{q-1}, & 0 \leq s \leq t \leq 1 \\ t^{q-1}(1-s)^{q-1}, & 0 \leq t \leq s \leq 1\end{cases}
$$


and

$$
g_{i}\left(\zeta_{i}, s\right)= \begin{cases}\zeta_{i}^{p_{i}+q-1}(1-s)^{q-1}-\left(\zeta_{i}-s\right)^{p_{i}+q-1}, & 0 \leq s \leq \zeta_{i}<1 \\ \zeta_{i}^{p_{i}+q-1}(1-s)^{q-1}, & 0<\zeta_{i} \leq s \leq 1\end{cases}
$$

Proof Applying Lemma 2.1, BVP (2.2) can be expressed as an equivalent integral equation

$$
u(t)=-\frac{1}{\Gamma(q)} \int_{0}^{t}(t-s)^{q-1} h(s) d s+C_{1} t^{q-1}+C_{2} t^{q-2}+\cdots+C_{n} t^{q-n}
$$

for $C_{i} \in \mathbb{R}(i=1,2, \ldots, n) \in \mathbb{R} . u^{(j)}(0)=0(j=0,1,2, \ldots, n-2)$ implies that $C_{2}=C_{3}=\cdots=$ $C_{n}=0$. Taking the Riemann-Liouville fractional integral of order $p_{i}>0$ for (2.6), we get

$$
\begin{aligned}
\left(I_{0+}^{p_{i}} u\right)(t) & =\int_{0}^{t} \frac{(t-s)^{p_{i}-1}}{\Gamma\left(p_{i}\right)}\left(C_{1} s^{q-1}-\int_{0}^{s} \frac{(s-r)^{q-1}}{\Gamma(q)} h(r) d r\right) d s \\
& =C_{1} \int_{0}^{t} \frac{(t-s)^{p_{i}-1} s^{q-1}}{\Gamma\left(p_{i}\right)} d s-\int_{0}^{t} \frac{(t-s)^{p_{i}-1}}{\Gamma\left(p_{i}\right)} \int_{0}^{s} \frac{(s-r)^{q-1}}{\Gamma(q)} h(r) d s d r \\
& =C_{1} \frac{t^{p_{i}+q-1} \Gamma(q)}{\Gamma\left(p_{i}+q\right)}-\frac{1}{\Gamma\left(p_{i}+q\right)} \int_{0}^{t}(t-s)^{p_{i}+q-1} h(s) d s .
\end{aligned}
$$

$u(1)=\sum_{i=1}^{m-2} \alpha_{i}\left(I_{0+}^{p_{i}} u\right)\left(\zeta_{i}\right)$ yields

$$
\begin{aligned}
C_{1} & -\int_{0}^{1} \frac{(1-s)^{q-1}}{\Gamma(q)} h(s) d s \\
& =C_{1} \sum_{i=1}^{m-2} \frac{\alpha_{i} \zeta_{i}^{p_{i}+q-1} \Gamma(q)}{\Gamma\left(p_{i}+q\right)}-\sum_{i=1}^{m-2} \frac{\alpha_{i}}{\Gamma\left(p_{i}+q\right)} \int_{0}^{\zeta_{i}}\left(\zeta_{i}-s\right)^{p_{i}+q-1} h(s) d s .
\end{aligned}
$$

Then we have

$$
C_{1}=\frac{1}{\Omega}\left[\int_{0}^{1} \frac{(1-s)^{q-1}}{\Gamma(q)} h(s) d s-\sum_{i=1}^{m-2} \frac{\alpha_{i}}{\Gamma\left(p_{i}+q\right)} \int_{0}^{\zeta_{i}}\left(\zeta_{i}-s\right)^{p_{i}+q-1} h(s) d s\right]
$$

Therefore, the unique solution of BVP (2.2) is written as

$$
\begin{aligned}
u(t)= & -\frac{1}{\Gamma(q)} \int_{0}^{t}(t-s)^{q-1} h(s) d s+\frac{1}{\Omega \Gamma(q)} \int_{0}^{1}(1-s)^{q-1} t^{q-1} h(s) d s \\
& -\frac{1}{\Omega} \sum_{i=1}^{m-2} \frac{\alpha_{i}}{\Gamma\left(p_{i}+q\right)} \int_{0}^{\zeta_{i}}\left(\zeta_{i}-s\right)^{p_{i}+q-1} t^{q-1} h(s) d s .
\end{aligned}
$$

Hence, by taking into account (2.1), we have

$$
\begin{aligned}
u(t)= & -\frac{1}{\Gamma(q)} \int_{0}^{t}(t-s)^{q-1} h(s) d s+\frac{1}{\Omega \Gamma(q)} \int_{0}^{1}(1-s)^{q-1} t^{q-1} h(s) d s \\
& -\frac{1}{\Omega} \sum_{i=1}^{m-2} \frac{\alpha_{i}}{\Gamma\left(p_{i}+q\right)} \int_{0}^{\zeta_{i}}\left(\zeta_{i}-s\right)^{p_{i}+q-1} t^{q-1} h(s) d s \\
& +\frac{1}{\Gamma(q)} \int_{0}^{1}(1-s)^{q-1} t^{q-1} h(s) d s-\frac{1}{\Gamma(q)} \int_{0}^{1}(1-s)^{q-1} t^{q-1} h(s) d s
\end{aligned}
$$




$$
\begin{aligned}
= & \frac{1}{\Gamma(q)} \int_{0}^{1}(1-s)^{q-1} t^{q-1} h(s) d s-\frac{1}{\Gamma(q)} \int_{0}^{t}(t-s)^{q-1} h(s) d s \\
& +\sum_{i=1}^{m-2} \frac{\alpha_{i} t^{q-1}}{\Omega \Gamma\left(p_{i}+q\right)}\left(\int_{0}^{1} \zeta_{i}^{p_{i}+q-1}(1-s)^{q-1} h(s) d s-\int_{0}^{\zeta_{i}}\left(\zeta_{i}-s\right)^{p_{i}+q-1} h(s) d s\right) \\
= & \int_{0}^{1} g(t, s) h(s) d s+\int_{0}^{1} \sum_{i=1}^{m-2} \frac{\alpha_{i} t^{q-1}}{\Omega \Gamma\left(p_{i}+q\right)} g_{i}\left(\zeta_{i}, s\right) h(s) d s \\
= & \int_{0}^{1} G(t, s) h(s) d s .
\end{aligned}
$$

The proof is complete.

Lemma 2.4 The Green's function $G(t, s)$ defined by (2.3)-(2.5) has the following properties:

(A1) $G(t, s) \in C([0,1] \times[0,1])$ and $G(t, s) \geq 0$, for all $t, s \in[0,1]$.

(A2) $G(t, s) \leq \max _{0 \leq t \leq 1} G(t, s) \leq G(s)$ and $G(t, s) \geq \min _{0 \leq t \leq 1} G(t, s) \geq \sigma(t) G(s)$, where

$$
G(s)=\frac{s(1-s)^{q-1}}{\Gamma(q-1)}+\sum_{i=1}^{m-2} \frac{\alpha_{i}}{\Omega \Gamma\left(p_{i}+q\right)} g_{i}\left(\zeta_{i}, s\right), \quad \sigma(t)=\frac{t^{q-1}(1-t)}{q-1}
$$

(A3) If $\theta \in(0,1 / 2)$, then $\min _{t \in[\theta, 1-\theta]} G(t, s) \geq \sigma(\theta) G(s)$.

Proof From the expression of $G(t, s)$, it is obvious that $G(t, s) \in C([0,1] \times[0,1])$. To prove $G(t, s) \geq 0$, we will show that $g(t, s), g_{i}\left(\zeta_{i}, s\right) \geq 0, i=1,2, \ldots, m-2$, for all $0 \leq s, t \leq 1$.

Let $k_{1}(t, s)=t^{q-1}(1-s)^{q-1}-(t-s)^{q-1}$ for $0 \leq s \leq t \leq 1$, then we have

$$
k_{1}(t, s)=(t-t s)^{q-1}-(t-s)^{q-1} \geq(t-s)^{q-1}-(t-s)^{q-1}=0 .
$$

For $0 \leq t \leq s \leq 1, k_{2}(t, s)=(t-t s)^{q-1} \geq 0$. Therefore, $g(t, s) \geq 0$ for all $0 \leq s, t \leq 1$. Now, let $k_{3}^{i}\left(\zeta_{i}, s\right)=\zeta_{i}^{p_{i}+q-1}(1-s)^{q-1}-\left(\zeta_{i}-s\right)^{p_{i}+q-1}$ for $0 \leq s \leq \zeta_{i}<1$, then we have

$$
\begin{aligned}
k_{3}^{i}\left(\zeta_{i}, s\right) & =\zeta_{i}^{p_{i}+q-1}(1-s)^{q-1}-\zeta_{i}^{p_{i}+q-1}\left(1-\frac{s}{\zeta}\right)^{p_{i}+q-1} \\
& \geq \zeta_{i}^{p_{i}+q-1}(1-s)^{q-1}-\zeta_{i}^{p_{i}+q-1}(1-s)^{p_{i}+q-1} \\
& =\zeta_{i}^{p_{i}+q-1}\left((1-s)^{q-1}-(1-s)^{p_{i}+q-1}\right) \geq 0 .
\end{aligned}
$$

For $0<\zeta_{i} \leq s \leq 1, k_{4}^{i}\left(\zeta_{i}, s\right)=\zeta_{i}^{p_{i}+q-1}(1-s)^{q-1} \geq 0$. Therefore $g_{i}\left(\zeta_{i}, s\right) \geq 0, i=1,2, \ldots, m-2$, for all $0 \leq s \leq 1$.

In the following, we prove (A2). When $s \leq t$, we have $1-s \geq 1-t$, then

$$
\begin{aligned}
k_{1}(t, s) & =t^{q-1}(1-s)^{q-1}-(t-s)^{q-1}=(q-1) \int_{t-s}^{t-t s} x^{q-2} d x \\
& \leq(q-1)(t-t s)^{q-2}((t-t s)-(t-s)) \\
& =(q-1) t^{q-2}(1-s)^{q-2}(1-t) s \\
& \leq(q-1) s(1-s)^{q-1}
\end{aligned}
$$


and

$$
\begin{aligned}
k_{1}(t, s) & =t^{q-1}(1-s)^{q-1}-(t-s)^{q-1} \\
& =(t-t s)^{q-2}(t-t s)-(t-s)^{q-2}(t-s) \\
& \geq(t-t s)^{q-2}(t-t s)-(t-t s)^{q-2}(t-s) \\
& =t^{q-2}(1-s)^{q-2}(1-t) s \\
& \geq t^{q-1}(1-t) s(1-s)^{q-1} .
\end{aligned}
$$

When $t \leq s$, we derive from $q>2$ that

$$
\begin{aligned}
k_{2}(t, s) & =t^{q-1}(1-s)^{q-1} \leq(q-1) t^{q-2} t(1-s)^{q-1} \\
& \leq(q-1) t^{q-2} s(1-s)^{q-1} \\
& \leq(q-1) s(1-s)^{q-1}
\end{aligned}
$$

and

$$
k_{2}(t, s)=t^{q-1}(1-s)^{q-1} \geq t^{q-1}(1-t) s(1-s)^{q-1} .
$$

Thus,

$$
\frac{t^{q-1}(1-t) s(1-s)^{q-1}}{\Gamma(q)} \leq g(t, s) \leq \frac{(q-1) s(1-s)^{q-1}}{\Gamma(q)}=\frac{s(1-s)^{q-1}}{\Gamma(q-1)} .
$$

From the above analysis, we have for $0 \leq s \leq 1$,

$$
\begin{aligned}
G(t, s) & \leq \max _{0 \leq t \leq 1} G(t, s)=\max _{0 \leq t \leq 1}\left(g(t, s)+\sum_{i=1}^{m-2} \frac{\alpha_{i} t^{q-1}}{\Omega \Gamma\left(p_{i}+q\right)} g_{i}\left(\zeta_{i}, s\right)\right) \\
& \leq \frac{s(1-s)^{q-1}}{\Gamma(q-1)}+\sum_{i=1}^{m-2} \frac{\alpha_{i}}{\Omega \Gamma\left(p_{i}+q\right)} g_{i}\left(\zeta_{i}, s\right)=G(s)
\end{aligned}
$$

and

$$
\begin{aligned}
G(t, s) & \geq \min _{0 \leq t \leq 1} G(t, s)=\min _{0 \leq t \leq 1}\left(g(t, s)+\sum_{i=1}^{m-2} \frac{\alpha_{i} t^{q-1}}{\Omega \Gamma\left(p_{i}+q\right)} g_{i}\left(\zeta_{i}, s\right)\right) \\
& \geq \frac{t^{q-1}(1-t) s(1-s)^{q-1}}{\Gamma(q)}+\sum_{i=1}^{m-2} \frac{\alpha_{i} t^{q-1}}{\Omega \Gamma\left(p_{i}+q\right)} g_{i}\left(\zeta_{i}, s\right) \\
& \geq \min _{0 \leq t \leq 1}\left\{\frac{t^{q-1}(1-t)}{q-1}, t^{q-1}\right\}\left(\frac{(q-1) s(1-s)^{q-1}}{\Gamma(q)}+\sum_{i=1}^{m-2} \frac{\alpha_{i}}{\Omega \Gamma\left(p_{i}+q\right)} g_{i}\left(\zeta_{i}, s\right)\right) \\
& =\frac{t^{q-1}(1-t)}{q-1} G(s)=\sigma(t) G(s) .
\end{aligned}
$$

Now we prove (A3). By (A1) and (A2), we have

$$
\min _{\theta \leq t \leq 1-\theta} G(t, s) \geq \min _{\theta \leq t \leq 1-\theta} \sigma(t) G(s)
$$


and

$$
\frac{\partial \sigma(t)}{\partial t}=\frac{(q-1) t^{q-2}(1-t)-t^{q-1}}{q-1}=\frac{(q-1) t^{q-2}-q t^{q-1}}{q-1}=\frac{t^{q-2}(q-1-q t)}{q-1} .
$$

It is clear that $\sigma(t)$ is increasing in $t \in\left[0, \frac{q-1}{q}\right]$ and decreasing in $t \in\left[\frac{q-1}{q}, 1\right]$, respectively. By $q>2$, we have

$$
\frac{q-1}{q}=1-\frac{1}{q}>1-\frac{1}{2}=\frac{1}{2}>\theta,
$$

thus, $\sigma(t)$ is increasing in $t \in\left[\theta, \frac{q-1}{q}\right]$, for $\frac{q-1}{q} \leq 1-\theta$ or $\frac{q-1}{q} \geq 1-\theta$, and we have $\min _{\theta \leq t \leq 1-\theta} \sigma(t)=\min \{\sigma(\theta), \sigma(1-\theta)\}$. For $0<\theta<1-\theta<1, q>2$, we get

$$
\sigma(\theta)-\sigma(1-\theta)=\frac{\theta^{q-1}(1-\theta)}{q-1}-\frac{(1-\theta)^{q-1} \theta}{q-1}=\frac{\theta^{q-1}(1-\theta)}{q-1}\left(1-\left(\frac{1-\theta}{\theta}\right)^{q-2}\right)<0 .
$$

Therefore

$$
\min _{\theta \leq t \leq 1-\theta} G(t, s) \geq \min _{\theta \leq t \leq 1-\theta} \sigma(t) G(s)=\sigma(\theta) G(s) .
$$

This proof is complete.

\section{Existence of multiple positive solutions}

In this section, we will consider the existence of multiple positive solutions for the BVP (1.2)-(1.3).

Let $E=\left\{u(t): u(t) \in C\left[-\tau_{1}, 1+\tau_{2}\right]\right\}$ denote a real Banach space with the norm $\|\cdot\|$ defined by $\|u\|=\max _{-\tau_{1} \leq t \leq 1+\tau_{2}}|u(t)|$. Define the cone $P \subset E$ by $P=\{u \in E: u(t) \geq 0\}$. Let

$$
\begin{aligned}
& K=\{u \in P: u(t) \geq \sigma(t)\|u\|, \forall t \in[0,1]\}, \\
& K_{r}=\{u \in K:\|u\|<r\}, \quad \partial K_{r}=\{u \in K:\|u\|=r\} .
\end{aligned}
$$

Suppose that $u(t)$ is a solution of (1.2)-(1.3); according to Lemma 2.3 and Remark 1.1, it can be written as

$$
u(t)= \begin{cases}u\left(\tau_{1}, t\right), & -\tau_{1} \leq t \leq 0 \\ \int_{0}^{1} G(t, s) f\left(s, u\left(s-\tau_{1}\right), u\left(s+\tau_{2}\right)\right) d s, & 0 \leq t \leq 1 \\ u\left(\tau_{2}, t\right), & 1 \leq t \leq 1+\tau_{2}\end{cases}
$$

where

$$
u\left(\tau_{1}, t\right)= \begin{cases}e^{\frac{a}{b} t}\left(\frac{1}{b} \int_{t}^{0} e^{-\frac{a}{b} s} \eta(s) d s+u(0)\right), & -\tau_{1} \leq t \leq 0, b \neq 0, \\ \frac{\eta(t)}{a}, & -\tau_{1} \leq t \leq 0, b=0\end{cases}
$$

and

$$
u\left(\tau_{2}, t\right)= \begin{cases}e^{-\frac{c}{d} t}\left(\frac{1}{d} \int_{1}^{t} e^{\frac{c}{d} s} \xi(s) d s+e^{\frac{c}{d}} u(1)\right), & 1 \leq t \leq 1+\tau_{2}, d \neq 0 \\ \frac{\xi(t)}{c}, & 1 \leq t \leq 1+\tau_{2}, d=0 .\end{cases}
$$


Throughout this paper, we assume that $v_{0}(t)$ is the solution of (1.2)-(1.3) with $f \equiv 0$. Clearly, $v_{0}(t)$ can be expressed as follows:

$$
v_{0}(t)= \begin{cases}v_{0}\left(\tau_{1}, t\right), & -\tau_{1} \leq t \leq 0 \\ 0, & 0 \leq t \leq 1 \\ v_{0}\left(\tau_{2}, t\right), & 1 \leq t \leq 1+\tau_{2}\end{cases}
$$

where

$$
v_{0}\left(\tau_{1}, t\right)= \begin{cases}\frac{e^{\frac{a}{b} t}}{b} \int_{t}^{0} e^{-\frac{a}{b} s} \eta(s) d s, & -\tau_{1} \leq t \leq 0, b \neq 0 \\ \frac{\eta(t)}{a}, & -\tau_{1} \leq t \leq 0, b=0\end{cases}
$$

and

$$
v_{0}\left(\tau_{2}, t\right)= \begin{cases}\frac{e^{-\frac{c}{d} t}}{d} \int_{1}^{t} e^{\frac{c}{d} s} \xi(s) d s, & 1 \leq t \leq 1+\tau_{2}, d \neq 0 \\ \frac{\xi(t)}{c}, & 1 \leq t \leq 1+\tau_{2}, d=0\end{cases}
$$

Obviously, $v_{0}(t) \geq 0$ for each $t \in\left[-\tau_{1}, 1+\tau_{2}\right]$.

Let $u(t)$ be a solution of (1.2)-(1.3) and $v(t)=u(t)-v_{0}(t)$. Noting that $v(t) \equiv u(t)$ for $0 \leq t \leq 1$, we have

$$
v(t)= \begin{cases}v\left(\tau_{1}, t\right), & -\tau_{1} \leq t \leq 0 \\ \int_{0}^{1} G(t, s) f\left(s,\left(v+v_{0}\right)\left(s-\tau_{1}\right),\left(v+v_{0}\right)\left(s+\tau_{2}\right)\right) d s, & 0 \leq t \leq 1 \\ v\left(\tau_{2}, t\right), & 1 \leq t \leq 1+\tau_{2}\end{cases}
$$

where

$$
\begin{aligned}
& \left(v+v_{0}\right)\left(s-\tau_{1}\right)=v\left(s-\tau_{1}\right)+v_{0}\left(s-\tau_{1}\right), \\
& \left(v+v_{0}\right)\left(s+\tau_{2}\right)=v\left(s+\tau_{2}\right)+v_{0}\left(s+\tau_{2}\right), \\
& v\left(\tau_{1}, t\right)= \begin{cases}e^{\frac{a}{b} t} v(0), & -\tau_{1} \leq t \leq 0, b \neq 0, \\
0, & -\tau_{1} \leq t \leq 0, b=0\end{cases}
\end{aligned}
$$

and

$$
v\left(\tau_{2}, t\right)= \begin{cases}e^{-\frac{c}{d}(t-1)} v(1), & 1 \leq t \leq 1+\tau_{2}, d \neq 0 \\ 0, & 1 \leq t \leq 1+\tau_{2}, d=0\end{cases}
$$

Define an operator $A: E \rightarrow E$ as follows:

$$
(A v)(t)= \begin{cases}\left(B_{1} v\right)(t), & -\tau_{1} \leq t \leq 0 \\ \int_{0}^{1} G(t, s) f\left(s,\left(v+v_{0}\right)\left(s-\tau_{1}\right),\left(v+v_{0}\right)\left(s+\tau_{2}\right)\right) d s, & 0 \leq t \leq 1 \\ \left(B_{2} v\right)(t), & 1 \leq t \leq 1+\tau_{2}\end{cases}
$$

where

$$
\left(B_{1} v\right)(t)= \begin{cases}e^{\frac{a}{b} t} \int_{0}^{1} G(0, s) f\left(s,\left(v+v_{0}\right)\left(s-\tau_{1}\right),\left(v+v_{0}\right)\left(s+\tau_{2}\right)\right) d s, & -\tau_{1} \leq t \leq 0, b \neq 0 \\ 0, & -\tau_{1} \leq t \leq 0, b=0\end{cases}
$$


and

$$
\left(B_{2} v\right)(t)= \begin{cases}e^{-\frac{c}{d}} \int_{0}^{1} f\left(s,\left(v+v_{0}\right)\left(s-\tau_{1}\right),\left(v+v_{0}\right)\left(s+\tau_{2}\right)\right) & \\ \times G(1, s) d s, & 1 \leq t \leq 1+\tau_{2}, d \neq 0 \\ 0, & 1 \leq t \leq 1+\tau_{2}, d=0\end{cases}
$$

It is easy to derive that $u$ is a positive solution of BVP (1.2)-(1.3) if $v=u-v_{0}$ is a nontrivial fixed point of $A: K \rightarrow K$, where $v_{0}$ is defined as before.

Lemma 3.1 $A: K \rightarrow K$ defined by (3.3) is completely continuous.

Proof For $v \in K$, we find from Lemma 2.4 and the definition of $A$ that $0 \leq(A v)(t) \leq$ $(A v)(0)$, for $t \in\left[-\tau_{1}, 0\right]$, and $0 \leq(A v)(t) \leq(A v)(1)$ for $t \in\left[1,1+\tau_{2}\right]$. Thus, $\|A v\|=\|A v\|_{[0,1]}=$ $\max _{0 \leq t \leq 1}|(A v)(t)|$. It follows from Lemma 2.4 that

$$
\begin{aligned}
(A v)(t) & \geq \sigma(t) \int_{0}^{1} G(s) f\left(s,\left(v+v_{0}\right)\left(s-\tau_{1}\right),\left(v+v_{0}\right)\left(s+\tau_{2}\right)\right) d s \\
& \geq \sigma(t)\|A v\|_{[0,1]}=\sigma(t)\|A v\| .
\end{aligned}
$$

Thus, $A(K) \subset K$. In addition, since $f$ is continuous, it follows that $A$ is continuous.

Let $Q_{1} \in K$ be bounded, that is, there exists a positive constant $M_{1}>0$ such that $\|v\|_{\left[-\tau_{1}, 1+\tau_{2}\right]} \leq M_{1}$ for all $v \in Q_{1}$. Then $\left\|v+v_{0}\right\|_{\left[-\tau_{1}, 1+\tau_{2}\right]} \leq M_{1}+M_{0} \triangleq M_{2}$ for $v \in Q_{1}$, where $v_{0}$ is defined as before. Define a set $Q_{2} \subset E$ as follows:

$$
Q_{2}=\left\{\psi \in E:\|\psi\|_{\left[-\tau_{1}, 1+\tau_{2}\right]} \leq M_{2}\right\}
$$

Hence, $\max _{-\tau_{1} \leq t \leq 1+\tau_{2}}\left|\left(v+v_{0}\right)(t)\right| \leq\left\|v+v_{0}\right\|_{\left[-\tau_{1}, 1+\tau_{2}\right]} \leq\|v\|_{\left[-\tau_{1}, 1+\tau_{2}\right]}+\left\|v_{0}\right\|_{\left[-\tau_{1}, 1+\tau_{2}\right]} \leq M_{2}$. Noting that $f$ is continuous on $[0,1] \times\left[0, M_{2}\right] \times\left[0, M_{2}\right]$, there exists a constant $M_{3}>0$ such that on $[0,1] \times\left[0, M_{2}\right] \times\left[0, M_{2}\right]$,

$$
\left|f\left(t,\left(v+v_{0}\right)\left(t-\tau_{1}\right),\left(v+v_{0}\right)\left(t+\tau_{2}\right)\right)\right| \leq M_{3}
$$

Therefore,

$$
\|A v\|_{\left[-\tau_{1}, 1+\tau_{2}\right]}=\|A v\|_{[0,1]} \leq M_{3} \int_{0}^{1} G(s) d s
$$

Hence, $A\left(Q_{1}\right)$ is bounded.

Finally, we show the operator $A$ is equicontinuous. For $v \in Q_{1}$, we have

$$
\begin{aligned}
& (A v)^{\prime}(t)=\int_{0}^{1} \frac{\partial}{\partial t} G(t, s) f\left(s,\left(v+v_{0}\right)\left(s-\tau_{1}\right),\left(v+v_{0}\right)\left(s+\tau_{2}\right)\right) d s, \quad t \in[0,1] \\
& (A v)^{\prime}(t)=\frac{a}{b} e^{\frac{a}{b} t} \int_{0}^{1} G(0, s) f\left(s,\left(v+v_{0}\right)\left(s-\tau_{1}\right),\left(v+v_{0}\right)\left(s+\tau_{2}\right)\right) d s, \quad t \in\left[-\tau_{1}, 0\right]
\end{aligned}
$$

and

$$
(A v)^{\prime}(t)=-\frac{c}{d} e^{-\frac{c}{d}(t-1)} \int_{0}^{1} G(1, s) f\left(s,\left(v+v_{0}\right)\left(s-\tau_{1}\right),\left(v+v_{0}\right)\left(s+\tau_{2}\right)\right) d s, \quad t \in\left[1,1+\tau_{2}\right]
$$


In the light of $f \leq M_{3}$ and

$$
\begin{aligned}
\frac{\partial}{\partial t} G(t, s) & \leq \frac{(q-1) t^{q-2}(1-s)^{q-1}+(q-1)(t-s)^{q-2}}{\Gamma(q)}+\sum_{i=1}^{m-2} \frac{\alpha_{i}(q-1) t^{q-2}}{\Omega \Gamma\left(p_{i}+q\right)} g_{i}\left(\zeta_{i}, s\right) \\
& \leq \frac{1}{\Gamma(q-1)}+\sum_{i=1}^{m-2} \frac{\alpha_{i}(q-1)}{\Omega \Gamma\left(p_{i}+q\right)} g_{i}\left(\zeta_{i}, s\right) \triangleq M^{\prime},
\end{aligned}
$$

we have $\left\|(A v)^{\prime}\right\| \leq M$ for some positive constant $M$. Thus, for $-\tau_{1} \leq t_{1}<t_{2} \leq 1+\tau_{2}$, we have

$$
\left\|(A v)\left(t_{2}\right)-(A v)\left(t_{1}\right)\right\| \leq \int_{t_{1}}^{t_{2}}\left|(A v)^{\prime}(s)\right| d s \leq M\left(t_{2}-t_{1}\right) \rightarrow 0, \quad \text { as } t_{1} \rightarrow t_{2} .
$$

Therefore, for any $\epsilon>0$, there exists $\delta=\delta(\epsilon)>0$ which is independent of $t_{1}, t_{2}$, and $v$ such that $\left\|(A v)\left(t_{2}\right)-(A v)\left(t_{1}\right)\right\| \leq \epsilon$, whenever $\left|t_{2}-t_{1}\right| \leq \delta$. Thus, $A\left(Q_{1}\right)$ is equicontinuous. In view of the Ascoli-Arzela theorem, we can easily see that $A: K \rightarrow K$ is a completely continuous operator. The proof is complete.

Further we make the following assumptions for $f(t, x, y)$ :

$\left(\mathrm{H}_{1}\right)$ There exists a constant $r_{1}>0$ such that $0 \leq x \leq r_{1}+\left\|v_{0}\right\|_{\left[-\tau_{1}, 0\right]}, 0 \leq y \leq r_{1}+\left\|v_{0}\right\|_{\left[1,1+\tau_{2}\right]}$, and $0 \leq t \leq 1$ implies $f(t, x, y)<\rho_{1} r_{1}$, where $\rho_{1}=\frac{1}{\int_{0}^{1} G(s) d s}$.

$\left(\mathrm{H}_{2}\right)$ There exists a constant $r_{2}>0$ such that $\sigma_{1} r_{2} \leq x \leq r_{2}, \sigma_{2} r_{2} \leq y \leq r_{2}$, and $\theta \leq t \leq$ $1-\theta$ implies $f(t, x, y)>\rho_{2} r_{2}$, where $\rho_{2}=\frac{1}{\sigma(\theta) \int_{\theta}^{1-\theta} G(s) d s}, \sigma_{1}=\min _{\theta-\tau_{1} \leq t \leq 1-\theta-\tau_{1}}|\sigma(t)|, \sigma_{2}=$ $\min _{\theta+\tau_{2} \leq t \leq 1-\theta+\tau_{2}}|\sigma(t)|$.

$\left(\mathrm{H}_{3}\right) f_{\infty}=\liminf _{x+y \rightarrow+\infty} \min _{t \in[0,1]} \frac{f(t, x, y)}{x+y}=\infty$.

$\left(\mathrm{H}_{4}\right) f^{\infty}=\limsup _{x+y \rightarrow+\infty} \max _{t \in[0,1]} \frac{f(t, x, y)}{x+y}=0$.

$\left(\mathrm{H}_{5}\right) f_{0}=\liminf _{x+y \rightarrow 0} \min _{t \in[0,1]} \frac{f(t, x, y)}{x+y}=\infty$.

$\left(\mathrm{H}_{6}\right) f^{0}=\limsup _{x+y \rightarrow 0} \max _{t \in[0,1]} \frac{f(t, x, y)}{x+y}=0$.

Theorem 3.1 Assume that $\left(\mathrm{H}_{1}\right),\left(\mathrm{H}_{2}\right)$, and $\left(\mathrm{H}_{3}\right)$ are satisfied. If $r_{1}>r_{2}>0$, then BVP (1.2)(1.3) has at least two positive solutions $u_{1}$ and $u_{2}$ such that

$$
0<r_{2}<\left\|u_{1}\right\|_{[0,1]}<r_{1}<\left\|u_{2}\right\|_{[0,1]} .
$$

Proof Let $A: K \rightarrow K$ be the cone preserving completely continuous that is defined by (3.3). Let $\Phi_{r_{1}}=\left\{v \in E:\|v\|<r_{1}\right\}$, then for any $v \in K \cap \partial \Phi_{r_{1}}$, we get

$$
\begin{aligned}
& 0 \leq\left(v+v_{0}\right)\left(t-\tau_{1}\right) \leq\|v\|+\left\|v_{0}\right\|_{\left[-\tau_{1}, 1\right]}=r_{1}+\left\|v_{0}\right\|_{\left[-\tau_{1}, 0\right]}, \quad 0 \leq t \leq 1, \\
& 0 \leq\left(v+v_{0}\right)\left(t+\tau_{2}\right) \leq\|v\|+\left\|v_{0}\right\|_{\left[0,1+\tau_{2}\right]}=r_{1}+\left\|v_{0}\right\|_{\left[1,1+\tau_{2}\right]}, \quad 0 \leq t \leq 1 .
\end{aligned}
$$

Thus, from (A3) and $\left(\mathrm{H}_{1}\right)$, we have

$$
\begin{aligned}
\|A v\| & =\|A v\|_{[0,1]}=\max _{t \in[0,1]}(A v)(t) \\
& =\max _{t \in[0,1]} \int_{0}^{1} G(t, s) f\left(s,\left(v+v_{0}\right)\left(s-\tau_{1}\right),\left(v+v_{0}\right)\left(s+\tau_{2}\right)\right) d s
\end{aligned}
$$




$$
\begin{aligned}
& \leq \int_{0}^{1} G(s) f\left(s,\left(v+v_{0}\right)\left(s-\tau_{1}\right),\left(v+v_{0}\right)\left(s+\tau_{2}\right)\right) d s \\
& <\rho_{1} r_{1} \int_{0}^{1} G(s) d s=r_{1}=\|v\| .
\end{aligned}
$$

Therefore,

$$
\|A v\|<\|v\|, \quad v \in K \cap \partial \Phi_{r_{1}} .
$$

Let $\Phi_{r_{2}}=\left\{v \in E:\|v\|<r_{2}\right\}$, then for any $v \in K \cap \partial \Phi_{r_{2}}$, we have

$$
\begin{aligned}
& r_{2}=\|v\| \geq v\left(t-\tau_{1}\right) \geq \sigma\left(t-\tau_{1}\right)\|v\| \geq \sigma_{1} r_{2}, \quad \theta \leq t \leq 1-\theta, \\
& r_{2}=\|v\| \geq v\left(t+\tau_{2}\right) \geq \sigma\left(t+\tau_{2}\right)\|v\| \geq \sigma_{2} r_{2}, \quad \theta \leq t \leq 1-\theta .
\end{aligned}
$$

Thus, from $\left(\mathrm{H}_{2}\right)$ and (A3) of Lemma 2.4, we get

$$
\begin{aligned}
\|A v\| & =\|A v\|_{[0,1]}=\max _{t \in[0,1]}(A v)(t) \\
& =\max _{t \in[0,1]} \int_{0}^{1} G(t, s) f\left(s,\left(v+v_{0}\right)\left(s-\tau_{1}\right),\left(v+v_{0}\right)\left(s+\tau_{2}\right)\right) d s \\
& \geq \min _{t \in[\theta, 1-\theta]} \int_{0}^{1} G(t, s) f\left(s,\left(v+v_{0}\right)\left(s-\tau_{1}\right),\left(v+v_{0}\right)\left(s+\tau_{2}\right)\right) d s \\
& \geq \sigma(\theta) \int_{0}^{1} G(s) f\left(s,\left(v+v_{0}\right)\left(s-\tau_{1}\right),\left(v+v_{0}\right)\left(s+\tau_{2}\right)\right) d s \\
& \geq \sigma(\theta) \int_{\theta}^{1-\theta} G(s) f\left(s,\left(v+v_{0}\right)\left(s-\tau_{1}\right),\left(v+v_{0}\right)\left(s+\tau_{2}\right)\right) d s \\
& =\sigma(\theta) \int_{\theta}^{1-\theta} G(s) f\left(s, v\left(s-\tau_{1}\right), v\left(s+\tau_{2}\right)\right) d s \\
& >\rho_{2} r_{2} \sigma(\theta) \int_{\theta}^{1-\theta} G(s) d s=r_{2}=\|v\| .
\end{aligned}
$$

So

$$
\|A v\|>\|v\|, \quad v \in K \cap \partial \Phi_{r_{2}} .
$$

Choose $L>0$ such that

$$
\left(\sigma_{1}+\sigma_{2}\right) L \sigma(\theta) \int_{\theta}^{1-\theta} G(s) d s>1 .
$$

From $\left(\mathrm{H}_{3}\right)$, there exists $R_{1}>0$ such that

$$
f(t, x, y) \geq L(x+y), \quad x, y \geq 0, x+y \geq R_{1}, 0 \leq t \leq 1
$$

Choose

$$
R_{0}>\max \left\{r_{1}, \frac{R_{1}}{2 \min \left\{\sigma_{1}, \sigma_{2}\right\}}\right\} .
$$


Let $\Phi_{R}=\left\{v \in E:\|v\|<R, R \geq R_{0}\right\}$, then for any $v \in K \cap \partial \Phi_{R}$, we have

$$
\begin{aligned}
& v\left(t-\tau_{1}\right) \geq \sigma\left(t-\tau_{1}\right)\|v\| \geq \sigma_{1} R \geq \min \left\{\sigma_{1}, \sigma_{2}\right\} R_{0}>\frac{1}{2} R_{1}, \quad \theta \leq t \leq 1-\theta, \\
& v\left(t+\tau_{2}\right) \geq \sigma\left(t+\tau_{2}\right)\|v\| \geq \sigma_{2} R \geq \min \left\{\sigma_{1}, \sigma_{2}\right\} R_{0}>\frac{1}{2} R_{1}, \quad \theta \leq t \leq 1-\theta .
\end{aligned}
$$

Then, from (3.6) and (3.7) we have

$$
\begin{aligned}
\|A v\| & =\|A v\|_{[0,1]}=\max _{t \in[0,1]}(A v)(t) \\
& =\max _{t \in[0,1]} \int_{0}^{1} G(t, s) f\left(s,\left(v+v_{0}\right)\left(s-\tau_{1}\right),\left(v+v_{0}\right)\left(s+\tau_{2}\right)\right) d s \\
& \geq \min _{t \in[\theta, 1-\theta]} \int_{0}^{1} G(t, s) f\left(s,\left(v+v_{0}\right)\left(s-\tau_{1}\right),\left(v+v_{0}\right)\left(s+\tau_{2}\right)\right) d s \\
& \geq \sigma(\theta) \int_{0}^{1} G(s) f\left(s,\left(v+v_{0}\right)\left(s-\tau_{1}\right),\left(v+v_{0}\right)\left(s+\tau_{2}\right)\right) d s \\
& \geq \sigma(\theta) \int_{\theta}^{1-\theta} G(s) f\left(s,\left(v+v_{0}\right)\left(s-\tau_{1}\right),\left(v+v_{0}\right)\left(s+\tau_{2}\right)\right) d s \\
& =\sigma(\theta) \int_{\theta}^{1-\theta} G(s) f\left(s, v\left(s-\tau_{1}\right), v\left(s+\tau_{2}\right)\right) d s \\
& \geq L \sigma(\theta) \int_{\theta}^{1-\theta} G(s)\left[v\left(s-\tau_{1}\right)+v\left(s+\tau_{2}\right)\right] d s \\
& \geq\left(\sigma_{1}+\sigma_{2}\right) R L \sigma(\theta) \int_{\theta}^{1-\theta} G(s) d s>R=\|v\| .
\end{aligned}
$$

Therefore,

$$
\|A v\|>\|v\|, \quad v \in K \cap \partial \Phi_{R} .
$$

Applying Lemma 2.2 to (3.4) and (3.5) yields the result that $A$ has a fixed point $v_{1} \in K \cap$ $\left(\bar{\Phi}_{r_{1}} \backslash \Phi_{r_{2}}\right)$ with $v_{1}(t) \geq \sigma_{1}\|u\|>0, t \in[0,1]$. Similarly, Lemma 2.2 associated with (3.4) and (3.8) shows that $A$ has another fixed point $v_{2} \in K \cap\left(\Phi_{R} \backslash \Phi_{r_{1}}\right)$ with $v_{2}(t) \geq \sigma_{2}\|u\|>0$, $t \in[0,1]$, which means that $u_{1}(t)=v_{1}(t)+v_{0}(t)$ and $u_{2}(t)=v_{2}(t)+v_{0}(t)$ are two positive solutions of BVP (1.2)-(1.3). Since

$$
\left\|u_{i}\right\|_{[0,1]}=\left\|v_{i}+v_{0}\right\|_{[0,1]}=\left\|v_{i}\right\|_{[0,1]}=\left\|A v_{i}\right\|_{[0,1]}=\left\|A v_{i}\right\|=\left\|v_{i}\right\|, \quad i=1,2,
$$

it follows that $u_{1}(t)$ and $u_{2}(t)$ satisfy

$$
0<r_{2}<\left\|u_{1}\right\|_{[0,1]}=\left\|v_{1}\right\|<r_{1}<\left\|v_{2}\right\|=\left\|u_{2}\right\|_{[0,1]} .
$$

The proof is complete.

Theorem 3.2 Assume that $\left(\mathrm{H}_{1}\right),\left(\mathrm{H}_{3}\right)$, and $\left(\mathrm{H}_{5}\right)$ are satisfied. There exist constants $R>r_{1}>$ $r>0$, then BVP (1.2)-(1.3) has at least two positive solutions $u_{1}$ and $u_{2}$ such that

$$
0<r<\left\|u_{1}\right\|_{[0,1]}<r_{1}<\left\|u_{2}\right\|_{[0,1]} .
$$


Proof Choose $0<r<r_{1}<R$, let $\Phi_{r}=\left\{v \in E:\|v\|<r, r<r_{1}\right\}$. For the same $L>0$ satisfying (3.6), $\left(\mathrm{H}_{5}\right)$ implies that

$$
f(t, x, y) \geq L(x+y), \quad x, y \geq 0, x+y \leq 2 r, 0 \leq t \leq 1 \text {. }
$$

Then for any $v \in K \cap \partial \Phi_{r}$, we have

$$
\begin{aligned}
& r=\|v\| \geq v\left(t-\tau_{1}\right) \geq \sigma\left(t-\tau_{1}\right)\|v\| \geq \sigma_{1} r, \quad \theta \leq t \leq 1-\theta, \\
& r=\|v\| \geq v\left(t+\tau_{2}\right) \geq \sigma\left(t+\tau_{2}\right)\|v\| \geq \sigma_{2} r, \quad \theta \leq t \leq 1-\theta .
\end{aligned}
$$

Then, from (3.6) and (3.9) we have

$$
\begin{aligned}
\|A v\| & =\|A v\|_{[0,1]}=\max _{t \in[0,1]}(A v)(t) \\
& =\max _{t \in[0,1]} \int_{0}^{1} G(t, s) f\left(s,\left(v+v_{0}\right)\left(s-\tau_{1}\right),\left(v+v_{0}\right)\left(s+\tau_{2}\right)\right) d s \\
& \geq \sigma(\theta) \int_{0}^{1} G(s) f\left(s,\left(v+v_{0}\right)\left(s-\tau_{1}\right),\left(v+v_{0}\right)\left(s+\tau_{2}\right)\right) d s \\
& \geq \sigma(\theta) \int_{\theta}^{1-\theta} G(s) f\left(s,\left(v+v_{0}\right)\left(s-\tau_{1}\right),\left(v+v_{0}\right)\left(s+\tau_{2}\right)\right) d s \\
& =\sigma(\theta) \int_{\theta}^{1-\theta} G(s) f\left(s, v\left(s-\tau_{1}\right), v\left(s+\tau_{2}\right)\right) d s \\
& \geq L \sigma(\theta) \int_{\theta}^{1-\theta} G(s)\left[v\left(s-\tau_{1}\right)+v\left(s+\tau_{2}\right)\right] d s \\
& \geq\left(\sigma_{1}+\sigma_{2}\right) r L \sigma(\theta) \int_{\theta}^{1-\theta} G(s) d s>r=\|v\| .
\end{aligned}
$$

Therefore,

$$
\|A v\|>\|v\|, \quad v \in K \cap \partial \Phi_{r} .
$$

Applying Lemma 2.2 to (3.4) and (3.10) yields that $A$ has a fixed point $v_{1} \in K \cap\left(\bar{\Phi}_{r_{1}} \backslash \Phi_{r}\right)$ with $v_{1}(t) \geq \sigma_{1}\|u\|>0, t \in[0,1]$. Similarly, Lemma 2.2 associated with (3.4) and (3.8) yields the result that $A$ has another fixed point $v_{2} \in K \cap\left(\Phi_{R} \backslash \Phi_{r_{1}}\right)$ with $v_{2}(t) \geq \sigma_{2}\|u\|>0, t \in$ $[0,1]$. This means that $u_{1}(t)=v_{1}(t)+v_{0}(t)$ and $u_{2}(t)=v_{2}(t)+v_{0}(t)$ are two positive solutions of BVP (1.2)-(1.3). Since

$$
\left\|u_{i}\right\|_{[0,1]}=\left\|v_{i}+v_{0}\right\|_{[0,1]}=\left\|v_{i}\right\|_{[0,1]}=\left\|A v_{i}\right\|_{[0,1]}=\left\|A v_{i}\right\|=\left\|v_{i}\right\|, \quad i=1,2,
$$

it follows that $u_{1}(t)$ and $u_{2}(t)$ satisfy

$$
0<r<\left\|u_{1}\right\|_{[0,1]}=\left\|v_{1}\right\|<r_{1}<\left\|v_{2}\right\|=\left\|u_{2}\right\|_{[0,1]} .
$$

The proof is complete. 
Theorem 3.3 Assume that $\left(\mathrm{H}_{2}\right),\left(\mathrm{H}_{4}\right)$, and $\left(\mathrm{H}_{6}\right)$ are satisfied. There exist constants $R>$ $r_{2}>r>0$, then BVP (1.2)-(1.3) has at least two positive solutions $u_{1}$ and $u_{2}$ such that

$$
0<r<\left\|u_{1}\right\|_{[0,1]}<r_{2}<\left\|u_{2}\right\|_{[0,1]} .
$$

Proof Choose $0<r<r_{2}<R$. By $\left(\mathrm{H}_{4}\right)$, for any $0<\varepsilon<\frac{1}{2 \int_{0}^{1} G(s) d s}$, there exists $R^{\prime}>0$ such that

$$
f(t, x, y) \leq \varepsilon(x+y), \quad x, y \geq 0, x+y \geq R^{\prime}, 0 \leq t \leq 1 .
$$

Putting

$$
C \triangleq \max _{0 \leq t \leq 1} \max _{0 \leq x, y, x+y \leq R^{\prime}}|f(t, x, y)|+1
$$

then

$$
f(t, x, y) \leq \varepsilon(x+y)+C, \quad x, y \geq 0,0 \leq t \leq 1 .
$$

Choose

$$
R_{0}>\left(C+2 \varepsilon\left\|v_{0}\right\|\right) \frac{\int_{0}^{1} G(s) d s}{1-2 \varepsilon \int_{0}^{1} G(s) d s} .
$$

Let $\Phi_{R}=\left\{v \in E:\|v\|<R, R \geq \max \left\{r_{2}, R_{0}\right\}\right\}$. Then, for any $v \in K \cap \partial \Phi_{R}$, we have

$$
\begin{aligned}
\|A v\| & =\|A v\|_{[0,1]}=\max _{t \in[0,1]}(A v)(t) \\
& =\max _{t \in[0,1]} \int_{0}^{1} G(t, s) f\left(s,\left(v+v_{0}\right)\left(s-\tau_{1}\right),\left(v+v_{0}\right)\left(s+\tau_{2}\right)\right) d s \\
& \leq \int_{0}^{1} G(s) f\left(s,\left(v+v_{0}\right)\left(s-\tau_{1}\right),\left(v+v_{0}\right)\left(s+\tau_{2}\right)\right) d s \\
& \leq \int_{0}^{1} G(s)\left[\varepsilon\left(\left(v+v_{0}\right)\left(s-\tau_{1}\right)+\left(v+v_{0}\right)\left(s+\tau_{2}\right)\right)+C\right] d s \\
& \leq \int_{0}^{1} G(s)\left[2 \varepsilon\left(\|v\|+\left\|v_{0}\right\|\right)+C\right] d s \\
& =2 \varepsilon R \int_{0}^{1} G(s) d s+\left(C+2 \varepsilon\left\|v_{0}\right\|\right) \int_{0}^{1} G(s) d s<R=\|v\| .
\end{aligned}
$$

Therefore,

$$
\|A v\|<\|v\|, \quad v \in K \cap \partial \Phi_{R} .
$$

Let $\Phi_{r}=\left\{v \in E:\|v\|<r, r<r_{2}\right\}$. By $\left(\mathrm{H}_{6}\right)$, for any $0<\varepsilon<\frac{1}{2 \int_{0}^{1} G(s) d s}$, there exists $0<r^{\prime}<r$ such that

$$
f(t, x, y) \leq \varepsilon(x+y), \quad x, y \geq 0, x+y<r^{\prime}, 0 \leq t \leq 1 .
$$


Putting

$$
C^{\prime} \triangleq \max _{0 \leq t \leq 1} \max _{0 \leq x, y, r^{\prime} \leq x+y \leq 2 r}|f(t, x, y)|+1
$$

then

$$
f(t, x, y) \leq \varepsilon(x+y)+C^{\prime}, \quad x, y \in \partial \Phi_{r}, 0 \leq t \leq 1 .
$$

Choose

$$
r>\left(C^{\prime}+2 \varepsilon\left\|v_{0}\right\|\right) \frac{\int_{0}^{1} G(s) d s}{1-2 \varepsilon \int_{0}^{1} G(s) d s} .
$$

Then, for any $v \in K \cap \partial \Phi_{r}$, we have

$$
\begin{aligned}
\|A v\| & =\|A v\|_{[0,1]}=\max _{t \in[0,1]}(A v)(t) \\
& =\max _{t \in[0,1]} \int_{0}^{1} G(t, s) f\left(s,\left(v+v_{0}\right)\left(s-\tau_{1}\right),\left(v+v_{0}\right)\left(s+\tau_{2}\right)\right) d s \\
& \leq \int_{0}^{1} G(s) f\left(s,\left(v+v_{0}\right)\left(s-\tau_{1}\right),\left(v+v_{0}\right)\left(s+\tau_{2}\right)\right) d s \\
& \leq \int_{0}^{1} G(s)\left[\varepsilon\left(\left(v+v_{0}\right)\left(s-\tau_{1}\right)+\left(v+v_{0}\right)\left(s+\tau_{2}\right)\right)+C^{\prime}\right] d s \\
& \leq \int_{0}^{1} G(s)\left[2 \varepsilon\left(\|v\|+\left\|v_{0}\right\|\right)+C^{\prime}\right] d s \\
& =2 \varepsilon r \int_{0}^{1} G(s) d s+\left(C^{\prime}+2 \varepsilon\left\|v_{0}\right\|\right) \int_{0}^{1} G(s) d s<r=\|v\| .
\end{aligned}
$$

So,

$$
\|A v\|<\|v\|, \quad v \in K \cap \partial \Phi_{r} .
$$

Applying Lemma 2.2 to (3.5) and (3.12) yields the result that $A$ has a fixed point $v_{1} \in K \cap$ $\left(\bar{\Phi}_{r_{1}} \backslash \Phi_{r}\right)$ with $v_{1}(t) \geq \sigma_{1}\|u\|>0, t \in[0,1]$. Similarly, from Lemma 2.2 associated with (3.5) and (3.11) one derives that $A$ has another fixed point $v_{2} \in K \cap\left(\bar{\Phi}_{R} \backslash \Phi_{r_{1}}\right)$ with $v_{2}(t) \geq$ $\sigma_{2}\|u\|>0, t \in[0,1]$. This means that $u_{1}(t)=v_{1}(t)+v_{0}(t)$ and $u_{2}(t)=v_{2}(t)+v_{0}(t)$ are two positive solutions of BVP (1.2)-(1.3). Since

$$
\left\|u_{i}\right\|_{[0,1]}=\left\|v_{i}+v_{0}\right\|_{[0,1]}=\left\|v_{i}\right\|_{[0,1]}=\left\|A v_{i}\right\|_{[0,1]}=\left\|A v_{i}\right\|=\left\|v_{i}\right\|, \quad i=1,2,
$$

it follows that $u_{1}(t)$ and $u_{2}(t)$ satisfy

$$
0<r<\left\|u_{1}\right\|_{[0,1]}=\left\|v_{1}\right\|<r_{2}<\left\|v_{2}\right\|=\left\|u_{2}\right\|_{[0,1]} .
$$

This completes the proof. 
We account for the control functions

$$
\begin{aligned}
& \qquad \begin{aligned}
\varphi(r) & =\max \left\{f(t, x, y):(t, x, y) \in[0,1] \times\left[0, r+r_{0}\right] \times\left[0, r+r_{0}^{\prime}\right]\right\}, \\
\psi(r) & =\min \left\{f(t, x, y):(t, x, y) \in[\theta, 1-\theta] \times\left[\sigma_{1} r, r\right] \times\left[\sigma_{2} r, r\right]\right\},
\end{aligned} \\
& \text { where } r_{0}=\left\|v_{0}\right\|_{\left[-\tau_{1}, 0\right]}, r_{0}^{\prime}=\left\|v_{0}\right\|_{\left[1,1+\tau_{2}\right]} .
\end{aligned}
$$

Theorem 3.4 Suppose that there exist two positive numbers $\xi_{2}<\xi_{1}$ such that one of the following conditions is satisfied:

(B) $\varphi\left(\xi_{1}\right)<\rho_{1} \xi_{1}, \psi\left(\xi_{2}\right)>\rho_{2} \xi_{2}$.

$\left(\mathrm{B}_{2}\right) \psi\left(\xi_{1}\right)>\rho_{2} \xi_{1}, \varphi\left(\xi_{2}\right)<\rho_{1} \xi_{2}$.

Then BVP (1.2)-(1.3) has at least one positive solution $u \in K$ such that

$$
\xi_{2}<\|u\|_{[0,1]}<\xi_{1}
$$

Proof Because of the similarity of the proof, we prove only this theorem under condition $\left(B_{1}\right)$. By assumption $\left(B_{1}\right)$, we have

$$
f(t, x, y) \leq \varphi\left(\xi_{1}\right)<\rho_{1} \xi_{1}, \quad f(t, x, y) \geq \psi\left(\xi_{2}\right)>\rho_{2} \xi_{2}
$$

which are the assumptions $\left(\mathrm{H}_{1}\right)$ and $\left(\mathrm{H}_{2}\right)$. By Theorem 3.1, we find that $A$ has a fixed point $v \in K \cap\left(\Phi_{\xi_{1}} \backslash \Phi_{\xi_{2}}\right)$, which means that (1.2)-(1.3) has at least one positive solution $u$ and $\xi_{2}<\|u\|_{[0,1]}<\xi_{1}$. This completes the proof.

Similarly, we can obtain the existence of multiple positive solutions for BVP (1.2)-(1.3).

Theorem 3.5 Suppose that there exist three positive numbers $\xi_{3}<\xi_{2}<\xi_{1}$ such that one of the following conditions is satisfied:

(B) $\varphi\left(\xi_{1}\right)<\rho_{1} \xi_{1}, \psi\left(\xi_{2}\right)>\rho_{2} \xi_{2}, \varphi\left(\xi_{3}\right)<\rho_{1} \xi_{3}$.

$\left(\mathrm{B}_{4}\right) \psi\left(\xi_{1}\right)>\rho_{2} \xi_{1}, \varphi\left(\xi_{2}\right)<\rho_{1} \xi_{2}, \psi\left(\xi_{3}\right)>\rho_{2} \xi_{3}$.

Then BVP (1.2)-(1.3) has at least two positive solutions $u_{1}, u_{2} \in K$ such that

$$
\xi_{3}<\left\|u_{1}\right\|_{[0,1]}<\xi_{2}<\left\|u_{2}\right\|_{[0,1]}<\xi_{1} .
$$

Theorem 3.6 Suppose that there exist four positive numbers $\xi_{4}<\xi_{3}<\xi_{2}<\xi_{1}$ such that one of the following conditions is satisfied:

(B⿱ $\left.\mathrm{B}_{5}\right) \varphi\left(\xi_{1}\right)<\rho_{1} \xi_{1}, \psi\left(\xi_{2}\right)>\rho_{2} \xi_{2}, \varphi\left(\xi_{3}\right)<\rho_{1} \xi_{3}, \psi\left(\xi_{4}\right)>\rho_{2} \xi_{4}$.

(B⿱ $\left.\mathrm{B}_{6}\right) \psi\left(\xi_{1}\right)>\rho_{2} \xi_{1}, \varphi\left(\xi_{2}\right)<\rho_{1} \xi_{2}, \psi\left(\xi_{3}\right)>\rho_{2} \xi_{3}, \varphi\left(\xi_{4}\right)<\rho_{1} \xi_{4}$.

Then BVP (1.2)-(1.3) has at least three positive solutions $u_{1}, u_{2}, u_{3} \in K$ such that

$$
\xi_{4}<\left\|u_{1}\right\|_{[0,1]}<\xi_{3}<\left\|u_{2}\right\|_{[0,1]}<\xi_{2}<\left\|u_{3}\right\|_{[0,1]}<\xi_{1}
$$

Theorem 3.7 Suppose that there exist $n+1$ positive numbers $\xi_{n+1}<\xi_{n}<\cdots<\xi_{2}<\xi_{1}$ such that one of the following conditions is satisfied: 
(B) $\varphi\left(\xi_{2 k-1}\right)<\rho_{1} \xi_{2 k-1}, \psi\left(\xi_{2 k}\right)>\rho_{2} \xi_{2 k}, k=1,2, \ldots,\left[\frac{n+2}{2}\right]$.

(B⿱ $\left.\mathrm{B}_{8}\right) \psi\left(\xi_{2 k-1}\right)>\rho_{2} \xi_{2 k-1}, \varphi\left(\xi_{2 k}\right)<\rho_{1} \xi_{2 k}, k=1,2, \ldots,\left[\frac{n+2}{2}\right]$.

Then BVP (1.2)-(1.3) has at least $n$ positive solutions $u_{i} \in K(i=1,2, \ldots, n)$ such that

$$
\xi_{i+1}<\left\|u_{i}\right\|_{[0,1]}<\xi_{i} .
$$

\section{Example}

Consider the following four-point BVP of delayed nonlinear fractional differential equations:

$$
\left\{\begin{array}{l}
D_{0+}^{\frac{5}{2}} u(t)+f\left(t, u\left(t-\frac{1}{6}\right), u\left(t+\frac{1}{9}\right)\right), \quad t \in[0,1] \\
u(0)=u^{\prime}(0)=0, \quad u(1)=8\left(I_{0+}^{\frac{1}{2}} u\right)\left(\frac{1}{4}\right)+5\left(I_{0+}^{\frac{3}{2}} u\right)\left(\frac{1}{2}\right)
\end{array}\right.
$$

where $f(t, x, y)=\frac{4\left(t^{2}+t+1\right)}{3}+\frac{x^{2}+y^{2}}{8}, 0 \leq t \leq 1, x, y \geq 0, q=5 / 2, \tau_{1}=\frac{1}{6}, \tau_{2}=\frac{1}{9}, \alpha_{1}=8, \alpha_{2}=5$, $p_{1}=\frac{1}{2}, p_{2}=\frac{3}{2}, \zeta_{1}=\frac{1}{4}, \zeta_{2}=\frac{1}{2}, m=4$. Choosing $\theta=\frac{1}{4}, r_{1}=2, r_{2}=\frac{1}{50}, r_{0}=r_{0}^{\prime}=\frac{1}{5}$.

By a simple calculation, we get

$$
\begin{aligned}
& \sigma_{1}=\min _{\frac{1}{12} \leq t \leq \frac{7}{12}} \sigma(t)=\sigma\left(\frac{1}{12}\right)=\frac{11 \sqrt{3}}{1,286}, \\
& \sigma_{2}=\min _{\frac{13}{36} \leq t \leq \frac{31}{36}} \sigma(t)=\sigma\left(\frac{31}{36}\right)=\frac{155 \sqrt{31}}{11,664}, \\
& \Omega=1-\sum_{i=1}^{2} \frac{\alpha_{i} \zeta_{i}^{p_{i}+q-1} \Gamma(q)}{\Gamma\left(p_{i}+q\right)} \approx 0.5827572 .
\end{aligned}
$$

By Lemmas 2.3 and 2.4 and the aid of a computer, we obtain

$$
\begin{aligned}
\rho_{1} & =\left(\int_{0}^{1} G(s) d s\right)^{-1}=\left(\int_{0}^{1} \frac{s(1-s)^{q-1}}{\Gamma(q-1)} d s+\sum_{i=1}^{m-2} \frac{\alpha_{i}}{\Omega \Gamma\left(p_{i}+q\right)} \int_{0}^{1} g_{i}\left(\zeta_{i}, s\right) d s\right)^{-1} \\
& =\left(\frac{q-1}{\Gamma(q+2)}+\sum_{i=1}^{m-2} \frac{\alpha_{i}}{\Omega \Gamma\left(p_{i}+q\right)}\left(\int_{0}^{1} \zeta_{i}^{p_{i}+q-1}(1-s)^{q-1} d s-\int_{0}^{\zeta_{i}}\left(\zeta_{i}-s\right)^{p_{i}+q-1} d s\right)\right)^{-1} \\
& =\left(\frac{q-1}{\Gamma(q+2)}+\sum_{i=1}^{m-2} \frac{\alpha_{i} \zeta_{i}^{p_{i}+q-1}\left(p_{i}+q\left(1-\zeta_{i}\right)\right)}{\Omega \Gamma\left(p_{i}+q+1\right) q}\right)^{-1} \\
& =\left(\frac{8}{35 \sqrt{\pi}}+\frac{19}{240 \Omega}+\frac{11}{384 \Omega}\right)^{-1} \approx 3.025561
\end{aligned}
$$

and

$$
\begin{aligned}
\rho_{2} & =\left(\sigma(\theta) \int_{\theta}^{1-\theta} G(s) d s\right)^{-1} \\
& =\left(\sigma\left(\frac{1}{4}\right)\right)^{-1}\left(\int_{\frac{1}{4}}^{\frac{3}{4}} \frac{s(1-s)^{q-1}}{\Gamma(q-1)} d s+\sum_{i=1}^{m-2} \frac{\alpha_{i}}{\Omega \Gamma\left(p_{i}+q\right)} \int_{\frac{1}{4}}^{\frac{3}{4}} g_{i}\left(\zeta_{i}, s\right) d s\right)^{-1} \\
& =16\left(\frac{117 \sqrt{3}-23}{1,120 \sqrt{\pi}}+\sum_{i=1}^{m-2} \frac{\alpha_{i}}{\Omega \Gamma\left(p_{i}+q\right)}\right.
\end{aligned}
$$




$$
\begin{aligned}
& \left.\times\left(\int_{\frac{1}{4}}^{\frac{3}{4}} \zeta_{i}^{p_{i}+q-1}(1-s)^{q-1} d s-\int_{\frac{1}{4}}^{\zeta_{i}}\left(\zeta_{i}-s\right)^{p_{i}+q-1} d s\right)\right)^{-1} \\
= & 16\left(\frac{117 \sqrt{3}-23}{1,120 \sqrt{\pi}}+\sum_{i=1}^{m-2} \frac{\alpha_{i}}{\Omega \Gamma\left(p_{i}+q\right)}\left[\frac{\zeta_{i}^{p_{i}+q-1}\left(3^{q}-1\right)}{q^{q}}-\frac{\left(\zeta_{i}-\frac{1}{4}\right)^{p_{i}+q}}{p_{i}+q}\right]\right)^{-1} \\
= & 16\left(\frac{117 \sqrt{3}-23}{1,120 \sqrt{\pi}}+\frac{9 \sqrt{3}-1}{320 \Omega}+\frac{72 \sqrt{3}-13}{6,144 \Omega}\right)^{-1} \approx 80.029634 .
\end{aligned}
$$

Therefore, for $(t, x, y) \in\left[\frac{1}{4}, \frac{3}{4}\right] \times\left[\frac{11 \sqrt{3}}{64,800}, \frac{1}{50}\right] \times\left[\frac{155 \sqrt{31}}{583,200}, \frac{1}{50}\right]$, we have

$$
\begin{aligned}
f(t, x, y) & =\frac{4\left(t^{2}+t+1\right)}{3}+\frac{x^{2}+y^{2}}{8} \geq f\left(\frac{1}{4}, \frac{11 \sqrt{3}}{64,800}, \frac{155 \sqrt{31}}{583,200}\right) \\
& >f\left(\frac{1}{4}, 0,0\right)=1.75>\rho_{2} r_{2} \approx 1.600593 .
\end{aligned}
$$

For $(t, x, y) \in[0,1] \times\left[0, \frac{11}{5}\right] \times\left[0, \frac{11}{5}\right]$, we obtain

$$
f(t, x, y)=\frac{4\left(t^{2}+t+1\right)}{3}+\frac{x^{2}+y^{2}}{8} \leq f\left(1, \frac{11}{5}, \frac{11}{5}\right)=5.21<\rho_{1} r_{1} \approx 6.051122
$$

and

$$
\begin{aligned}
f_{\infty} & =\liminf _{x+y \rightarrow+\infty} \min _{t \in[0,1]} \frac{f(t, x, y)}{x+y} \\
& =\liminf _{x+y \rightarrow+\infty} \min _{t \in[0,1]} \frac{1}{x+y}\left(\frac{4\left(t^{2}+t+1\right)}{3}+\frac{x^{2}+y^{2}}{8}\right) \\
& =\liminf _{x+y \rightarrow+\infty} \frac{1}{x+y}\left(\frac{4}{3}+\frac{x^{2}+y^{2}}{8}\right) \\
& \geq \liminf _{x+y \rightarrow+\infty} \frac{1}{x+y}\left(\frac{4}{3}+\frac{(x+y)^{2}}{16}\right)=\infty
\end{aligned}
$$

Thus $\left(\mathrm{H}_{1}\right)-\left(\mathrm{H}_{3}\right)$ hold. With the use of Theorem 3.1, BVP (4.1) has at least two positive solutions $u_{1}$ and $u_{2}$ such that $0<\frac{1}{50}<\left\|u_{1}\right\|_{[0,1]}<2<\left\|u_{2}\right\|_{[0,1]}$.

\section{Competing interests}

The authors declare that they have no competing interests.

\section{Authors' contributions}

All authors contributed equally and significantly in writing this paper. All authors read and approved the final manuscript.

\section{Acknowledgements}

The authors would like to thank the anonymous referees for their useful and valuable suggestions. This work was supported by the National Natural Sciences Foundation of Peoples Republic of China under Grant (No. 11161025), Yunnan Province natural scientific research fund project (No. 2011FZ058).

Received: 25 June 2014 Accepted: 30 December 2014 Published online: 31 January 2015

\section{References}

1. Babakhani, A, Gejji, V: Existence of positive solutions of nonlinear fractional differential equations. J. Math. Anal. Appl. 278, 434-442 (2003)

2. Lakshmikantham, V, Leela, S: Nagumo-type uniqueness result for fractional differential equations. Nonlinear Anal. 71, 2886-2889 (2009)

3. Feng, $M, G e, W$ : Existence results for a class of $n$th order $m$-point boundary value problems in Banach spaces. Appl. Math. Lett. 22, 1303-1308 (2009) 
4. Chang, Y-K, Nieto, JJ: Some new existence results for fractional differential inclusions with boundary conditions. Math. Comput. Model. 49, 605-609 (2009)

5. Ahmad, B, Nieto, JJ: Existence results for nonlinear boundary value problems of fractional integrodifferential equations with integral boundary conditions. Bound. Value Probl. 2009, Article ID 708576 (2009)

6. Abbas, S: Existence of solutions to fractional order ordinary and delay differential equations and applications. Electron. J. Differ. Equ. 2011, 9 (2011)

7. Bai, Z: On positive solutions of a nonlocal fractional boundary value problem. Nonlinear Anal. 72, 916-924 (2010)

8. Bai, C, Ma, J: Eigenvalue criteria for existence of multiple positive solutions to boundary value problems of second-order delay differential equations. J. Math. Anal. Appl. 301(2), 457-476 (2005)

9. Hong, $\mathrm{CH}$, Yeh, CC, Lee, CF: Existence of positive solutions for functional differential equations. Comput. Math. Appl. 40(6), 783-792 (2000)

10. Anguraja, A, Latha Maheswari, M: Existence of solutions for fractional impulsive neutral functional infinite delay integro-differential equations with nonlocal conditions. J. Nonlinear Sci. Appl. 5, 271-280 (2012)

11. Tariboon, J, Ntouyas, SK, Sudsutad, W: Positive solutions for fractional differential equations with three-point multi-term fractional integral boundary conditions. Adv. Differ. Equ. 2014, 28 (2014)

12. Zhang, $X Q$, Wang, L, Sun, Q: Existence of positive solutions for a class of nonlinear fractional differential equations with integral boundary conditions and a parameter. Appl. Math. Comput. 226, 708-718 (2014)

13. Benchohra, M, Henderson, J, Ntouyas, SK, Ouahab, A: Existence results for fractional order functional differential equations with infinite delay. J. Math. Anal. Appl. 338, 1340-1350 (2008)

14. Liao, C, Ye, H: Existence of positive solutions of nonlinear fractional delay differential equations. Positivity 13, 601-609 (2009)

15. Goodrich, C: Existence of a positive solution to a class of fractional differential equations. Appl. Math. Lett. 23 1050-1055 (2010)

16. Zhao, Y, Sun, S, Han, Z, Li, Q: The existence of multiple positive solutions for boundary value problems of nonlinear fractional differential equations. Commun. Nonlinear Sci. Numer. Simul. 16(4), 2086-2097 (2011)

17. Bai, Z, Lv, H: Positive solutions for boundary value problem of nonlinear fractional differential equation. J. Math. Anal. Appl. 311, 495-505 (2005)

18. Zhao, D, Liu, Y: Multiple positive solutions for nonlinear fractional boundary value problems. Sci. World J. 2013, Article ID 473828 (2013)

19. $\mathrm{Xu}, \mathrm{X}$, Jiang, $\mathrm{D}$, Yuan, C: Multiple positive solutions for the boundary value problem of a nonlinear fractional differential equation. Nonlinear Anal., Theory Methods Appl. 71(10), 4676-4688 (2009)

20. Jiang, J, Liu, L, Wu, Y: Multiple positive solutions of singular fractional differential system involving Stieltjes integral conditions. Electron. J. Qual. Theory Differ. Equ. 2012, 43 (2012)

21. Zhao, KH, Gong, P: Existence of positive solutions for a class of higher-order Caputo fractional differential equation. In Qualitative Theory of Dynamical Systems (2014). doi:10.1007/s12346-014-0121-0

22. Zhao, KH, Gong, P: Positive solutions of Riemann-Stieltjes integral boundary problems for the nonlinear coupling system involving fractional-order differential. Adv. Differ. Equ. 2014, 254 (2014)

23. Zhao, KH, Gong, P: Positive solutions for impulsive fractional differential equations with generalized periodic boundary value conditions. Adv. Differ. Equ. 2014, 255 (2014)

24. Kilbas, AA, Srivastava, HM, Trujillo, JJ: Theory and Applications of Fractional Differential Equations. North-Holland Mathematics Studies, vol. 204. Elsevier, Amsterdam (2006)

25. Podlubny, I: Fractional Differential Equations. Academic Press, New York (1993)

26. Guo, D, Lakshmikantham, V, Liu, X: Nonlinear Integral Equations in Abstract Spaces. Mathematics and Its Applications, vol. 373. Kluwer Academic, Dordrecht (1996)

\section{Submit your manuscript to a SpringerOpen ${ }^{\circ}$ journal and benefit from:}

- Convenient online submission

Rigorous peer review

- Immediate publication on acceptance

- Open access: articles freely available online

- High visibility within the field

- Retaining the copyright to your article 